\title{
Construcción y validación de un cuestionario de evaluación al desempeño docente mediante Análisis Factorial Exploratorio
}

\author{
Tonys Romero Díaz ${ }^{1}$ \\ Almudena Martínez Gimeno ${ }^{2}$
}

\section{RESUMEN}

El artículo presenta la metodología seguida para analizar la estructura de un cuestionario de evaluación al desempeño docente, realizado en la Facultad Regional Multidisciplinaria de Chontales, de la UNAN-MANAGUA. El objetivo del estudio fue determinar las dimensiones y sus índices de fiabilidad, en que el trabajo docente puede dividirse, para luego incorporar estas variables a los procesos de evaluación del desempeño docente en la facultad. Se retoman los principales aportes de cada uno de los actores más representativos en este campo de estudio, donde todos coinciden que la práctica docente es una actividad multidimensional. El estudio fue descriptivo y correlacional, se utilizó un muestreo multietápico. La técnica de análisis corresponde al Análisis Factorial Exploratorio, usando el método de Mínimos Cuadrados No ponderados. Los resultados indican que las veinticinco preguntas se agrupan en tres dimensiones, las cuales explican el 63.1

$\%$ de la varianza, y que la correlación entre estas dimensiones es alta. Así mismo, los índices de fiabilidad de cada una de ellas son superiores a 0.85 , y de 0.96 para todo el cuestionario. Por tanto se concluye que la estructura final de cuestionario es muy fiable para utilizarse en el proceso de evaluación al desempeño docente de la Facultad.

Palabras claves: Evaluación, desempeño docente, análisis factorial, cuestionario, fiabilidad.

Recibido: 27 de febrero de 2017

Aceptado: 05 de junio de 2017

1 Doctor en Educación e Intervención Social. Profesor de Matemática y Estadística de la Universidad Nacional Autónoma de Nicaragua, Managua. Departamento de Ciencias de Educación y Humanidades. UNAN-Managua, FAREM Chontales. Correo: tonyromerong@hotmail.com

2 Doctora en Didáctica y Organización de Instituciones Educativas. Profesora del Departamento de Educación y Psicología Social de la Universidad Pablo Olavide, de Sevilla. Correo: amartinez@upo.es 


\title{
Elaboration and validation of an evaluation questionnaire to teacher performance through Exploratory Factor Analysis
}

\begin{abstract}
This article presents the methodology used to analyze the structure of an evaluation questionnaire to the teaching performance, carried out at the Regional Multidisciplinary Faculty of Chontales, UNAN-MANAGUA. The objective of the study was to determine the dimensions and their reliability indexes, in which the teaching work can be divided, and then incorporate these variables to the process of evaluation of teaching performance in the faculty. The main contributions of each of the most representative actors in this field of study are taken up, where everyone agrees that teaching practice is a multidimensional activity. The study was descriptive and correlational, and featured multistage sampling. The analysis technique corresponds to the Exploratory Factor Analysis, using the Unweighted Least Squares method. The results indicate that the twenty-five questions are grouped in three dimensions, which explain $63.1 \%$ of the variance, and that the correlation between these dimensions is high. Likewise, the reliability indexes of each of them are superior to 0.85 , and of 0.96 for the whole questionnaire. Therefore it is concluded that the final structure of questionnaire is very reliable to be used in the evaluation process to the faculty's teaching performance.
\end{abstract}

Keywords: Evaluation, teacher performance, factor analysis, questionnaire, reliability. 


\section{INTRODUCCIÓN}

La evaluación del profesorado en nuestro contexto ha adquirido mucha relevancia en estos tiempos, tal así que las máximas autoridades de la UNAN-MANAGUA han expresado públicamente la necesidad de evaluar el trabajo que realizan los docentes dentro y fuera del aula de clases. La gran cantidad de tiempo y recursos, que se le dedican a la docencia en nuestra institución cada vez cobra mayor importancia, lo que implica que la sociedad demanda mejores resultados en el proceso, sin duda alguna, el tema constituye un desafío para la Universidad al incorporarse en la planificación e investigación.

En esta ocasión se presentan los resultados obtenidos de la estructura de un cuestionario para evaluar la docencia (Romero Díaz y Martínez, 2017), después de haber aplicado un Análisis Factorial Exploratorio, 1o que ha permitió comprobar la multidimensionalidad del mismo, pues tal como lo señalan Marsh (1984), Mateo (1988), García Ramos (1996), Morón (1999) y Fernández (2008), la docencia es una actividad compleja e implica el manejo de varios factores simultáneamente del docente.

Es cierto que se realizan muchos esfuerzos por mejorar la práctica docente, sin embargo, la cultura de la evaluación al docente es vista con cierta temeridad por parte de todos los involucrados, y aún no termina de transformar todas las labores cotidianas de la educación superior.

Dentro de las principales razones por las que la evaluación al desempeño debe comenzar a despuntar en nuestra Universidad, tenemos la transformación curricular iniciada en el 2013 como principal motor de impulso, dado que debe verse como el comienzo de una revolución en beneficio de la misma. También, en múltiples reuniones, asambleas, consejos académicos y otros espacios se ha planteado la necesidad de disponer de mecanismos que aseguren la calidad del trabajo desempeñado por los docentes.
Actualmente muchos docentes cuando escuchan la palabra evaluación a su desempeño, piensan que es debido a una mala práctica en su trabajo, la cultura de la evaluación al trabajo académico en el contexto nicaragüense es muy escasa, porque se piensa que sólo se debe de estudiar el comportamiento de aquellos docentes que entregan malos resultados en los exámenes finales o en el rendimiento general. No se valora todo el proceso del trabajo frente al alumnado, únicamente se tiene una idea del trabajo que se realiza por los resultados en las calificaciones o informes finales de la asignatura.

El modelo de evaluación para el desempeño docente en nuestra universidad aún no está definido, aunque en el reglamento interno de los docentes existen artículos de las funciones y la manera en cómo se deben cumplir las mismas, no es específico en la metodología a seguir para valorar si el trabajo realizado cumple o no con lo establecido en el reglamento, del mismo modo no se detalla que debe hacerse cuando el desempeño de un docente no es aceptable.

Dado el contexto actual en Nicaragua, referido a la evaluación y acreditación de las carreras en universidades por el CNEA (Comisión Nacional de Evaluación y Acreditación), es necesario que la universidad disponga de mecanismos e instrumentos para evaluar internamente y sistemáticamente el trabajo académico que realizan los docentes. ¿Por qué es necesaria esta evaluación? Veamos como Piaget opina al respecto: "Las mejores reformas educativas fracasarán si no se dispone de maestros en calidad y número suficiente" (Piaget, 1969).

El anterior rector de esta Alma Mater, Elmer Cisneros se refirió a la evaluación de las universidades como el control de calidad que una fábrica lleva a diario en sus procesos de manufactura; refirió que no es posible acercarnos a la calidad de la educación sino revisamos constantemente nuestra práctica docente en la institución mediante la evaluación y autoevaluación, por tanto, estos procesos serán los que nos certifiquen 
que lo que hacemos cumple con lo que la sociedad nos demanda.

Muchos docentes plantean la necesidad de que el maestro sea evaluado porque eso mismo se hace a nuestros estudiantes. Así, muchos docentes y coordinadores de carrera han empezado a utilizar cuestionarios para evaluar la práctica docente en las clases magistrales e individuales, lo cual indica que la cultura de evaluación del profesorado empieza a ser utilizada sistemáticamente en nuestra universidad.

Elobjetivo principal de estainvestigación se fundamentó en determinar las variables de la práctica docente más relevantes de la evaluación al desempeño docente, así como determinar las dimensiones latentes evaluadas que garanticen índices aceptables de fiabilidad de en cada una de ellas y en todo el cuestionario.

\section{Aproximaciones al concepto de evaluación del desempeño docente}

En nuestro contexto, las aproximaciones que más se asemejan a evaluar la docencia son vertidas por Rueda (2011) quién ha realizado muchos ejercicios similares en México. Chile es otro referente para análisis de la temática, donde se llevan a cabo ejercicios de evaluación docente, tanto en el nivel secundario como en la educación superior (Urriola, 2013; Zambrano, 2014).

Cronbach (1963) y Stufflebeam (1995) coinciden que la evaluación al desempeño docente es el proceso planificado de recogida de datos para revisar que se cumplen los objetivos de la práctica docente mediante el cual se ayuda a la reflexión y toma de decisiones, en esta definición vemos que se mantienen elementos de la evaluación propuesta por Tyler (1969). En cambio Valdés (2000) añade a este proceso el cumplimiento de las funciones determinadas por el contexto y a las que son inherentes a la profesión docente.
Sin duda alguna, las aproximaciones anteriores pueden resumirse en la exposición que hace Dias (2000), quien argumenta que la evaluación al desempeño docente es un conjunto de acciones organizadas y planificadas para valorar la interacción docente, estudiante y entorno, donde el objetivo es favorecer el proceso de enseñanza aprendizaje, así como el mejoramiento personal e institucional. Como tal no es un fin en sí mismo, apenas una herramienta para la mejora del trabajo y la buena toma de decisiones e implementar cambios para superar las debilidades encontradas. Este concepto lo consideraremos sinónimo de evaluación del profesorado, puesto que en otros contextos puede hallarse referida así.

Remontándonos a los primeros indicios sobre la temática, hemos de encontrar siempre que la referencia del tema fue el contexto anglosajón, algunos de los primeros en estudiar la evaluación docente fueron Boyce, Emans y Barr; muchos de los trabajo que desarrollaron no fueron sistematizadas, Salazar (2008), destaca a Renmers como el padre de la evaluación docente quien desarrolló numerosos estudios entre los que señala que si más de 25 estudiantes evalúan los profesores mediante un test, esta medida es tan fiable que puede emplearse para predecir el éxito de los profesores.

La literatura muestra que a partir de la década de los sesenta se realizaron numerosos estudios sobre esta problemática. Salazar (2008), destaca que Simpson en 1965 en su libro "Teacherself-evaluation" considera la autoevaluación como un factor principal para el perfeccionamiento docente, sin duda alguna en esta obra se destaca la autoevaluación como uno de los editores del comportamiento docente y una de las estrategias que más pueden incidir en su eficacia docente. El mismo autor señala que en 1970, Valdman y Peck dieron a conocer los resultados sobre las características de los mejores profesores según como lo conciben los alumnos, estas características pueden agruparse en cinco grandes dimensiones: cordialidad 
y optimismo, equilibrio emocional y dominio de la materia, actividad y dinamismo, control o disciplina y actitud democrática. Similar trabajo realizó Morón (1999) en su tesis doctoral, donde los resultados tienen muchas similitudes.

\section{Modelos de evaluación al desempeño profesional docente}

Viana (2000) establece que la palabra modelo se utiliza con dos sentidos: "prescriptivo" donde se destaca la metodología así como el valor particular que se le da a los datos; y el "descriptivo" que es el conjunto de afirmaciones encontradas y su generalización.

Del mismo modo, Stake (1982), planteó dos modelos grandes para la evaluación: la evaluación cualitativa, que está relacionado con el argumento convertido en singular, donde se identifican eventos y estudios de casos y las variables no interesan tanto en su control o medición como en los factores que explican su naturaleza. Mientras que en la evaluación cuantitativa se identifican las variables, tienen mucha importancia y se interpretan tratando de generalizar. En este sentido, podríamos confundir evaluación con investigación porque ambos están tratando de cumplir un mismo propósito en la estructuración de nuevos conocimientos a través de los fenómenos estudiados desde diferentes perspectivas.

Una característica principal que debe tener todo proceso de evaluación es la objetividad, ya que debemos evitar el sesgo que marca el subjetivismo de quienes evalúan. Una evaluación bien entendida y desarrollada en el seno de la planificación universitaria nos permite cumplir con objetivos para desarrollar el proceso final para la retroalimentación del proceso educativo y permite ser capaces de mostrar la veracidad de la información al momento de emitir un juicio en particular.

Una gran cantidad de investigaciones aportan los objetivos que debe tomar en cuenta la evaluación del profesorado, Mateo (1998) destaca seis categorías:
- Conocimiento docente: está referida a la preparación del profesor en las materias relacionadas a su especialidad.

- Destrezas docentes: se refiere a los medio que utilizan los profesores para preparar su docencia, es decir, los métodos, técnicas, didáctica, estímulos, etc.

- Competencia docente: es la preparación específica del profesor para cumplir con sus responsabilidades establecidas en el trabajo tales como el diseño y planificación docente.

- Efectividad docente: está relacionada con la contribución que hacen los profesores a los logros obtenidos por los estudiantes dentro de un área específica de contenidos.

- Productividad docente: es similar al factor de efectividad docente, sólo que acá es valorado el ámbito formativo y no el sumativo.

- Profesionalidad docente: es el objeto más importante de estudio de la evaluación docente porque se refiere al reconocimiento verdadero que debe tener un profesor como profesional de la docencia, estas conductas van con el cumplimiento profesional, integración, autocrítica, etc.

- Otra aportación importante al objeto y propósito de la evaluación docente tiene como referencia a un clásico en evaluación, Scriven (1994), detalla cuatro aspectos que dan sustento al objeto de la evaluación del profesorado, el contenido de estos aspectos es el siguiente:

- Conocimiento de la materia: tanto en las áreas de su competencia como en las materias transversales del currículum.

- Competencias instruccionales: destacándose las destrezas comunicativas, de gestión, de programación, desarrollo, materiales del curso y evaluación de la asignatura.

- Competencias evaluativas: el docente debe tener conocimiento sobre la evaluación de los estudiantes, tanto en la construcción, administración, calificación, registros e informes del rendimiento de los estudiantes. 
- Profesionalidad: el profesor debe tener ética, actitud, vocación de servicio, actitud de mejora; debe tener conocimiento sobre su profesión y de poder ayudar a los demás docentes tanto en trabajos operacionales como en la investigación sobre la enseñanza.

\section{MATERIALES Y MÉTODOS}

La metodología seguida en esta investigación ha sido meramente cuantitativa, ya que se usa la recolección de datos para probar hipótesis en base a mediciones numéricas, donde el análisis estadístico fue indispensable para establecer patrones y probar teorías (Hernández Sampieri, Collado y Baptista, 2014). Se utilizó un diseño no experimental porque se realizó sin la manipulación deliberada de variables, y sólo se observaron los fenómenos en su ambiente natural para después analizarlos. De este tipo de diseño recurrimos al diseño transeccional que evalúa una situación o determina la relación entre un conjunto de variables en un momento específico del contexto. La profundidad alcanzada en el estudio fue descriptiva y correlacional.

La población en estudio estuvo compuesta por 3138 estudiantes, que es la matrícula de la Facultad Regional Multidisciplinaria de Chontales, de la cual se tomó una muestra de 773 estudiantes. Se ha optado por un muestreo polietápico (multietápico o de etapas múltiples) el cual implica la selección de la muestra en diferentes pasos, es decir, se combinaron varios tipos de muestreos simultáneamente (Pérez, 2005).

Para ello, se estratificó la muestra en un primer momento para que estuvieran representados todos los turnos y modalidades. Luego en el segundo momento, se seleccionaron muestras estratificadas en cada uno de las carreras, para luego seleccionar los años a los que se iba a llegar usando el muestreo por conglomerado. Finalmente se seleccionaron muestras sistemáticas de estudiantes en cada sección visitada. El muestreo multiétapico consiste en la selección de unidades muestrales mayores hasta conseguir la unidad muestra más pequeña, y en el mismo puede existir una combinación de los diferentes tipos de muestreo probabilísticos (Cochran, 1977).

Se ha empleado el cuestionario como técnica de recolección de la información, mismo que fue construido tomando en cuenta un diagnóstico previo (Romero Díaz y Martínez, 2017). Tal y como indican Barroso y Cabero (2010:38) "posiblemente nos encontramos ante uno de los instrumentos más ampliamente utilizado en la investigación educativa". Ello es debido fundamentalmente a su facilidad de uso y su carácter directo (Hayman, 1984). Travers (1986), señala que es un instrumento destinado a determinar la naturaleza de un estado de cosas existentes; además, su carácter impersonal, la estandarización del vocabulario empleado, la concisión de las preguntas, etc., permiten suponer una cierta uniformidad respecto a las respuestas obtenidas.

\section{Técnica de análisis}

Se utilizó el Análisis Factorial Exploratorio (AFE) como técnicas del análisis multivariado, la cual es la más recomendada y usada por investigadores para reducir dimensiones y encontrar la estructura adecuada de un cuestionario (Valderrey, 2010). El AFE permitió descubrir el número adecuado de dimensiones y la distribución de los veintiocho ítems. En la revisión de este tipo técnica en otros estudios similares, se ha encontrado que en más del $50 \%$ de estos trabajos se han encontrado entre cinco y diez dimensiones, por lo que la mayoría de las investigaciones sugieren que el ejercicio docente es una práctica multidimensional.

El análisis factorial es una técnica de análisis multivariante que sirve para identificar las dimensiones subyacentes en un cuestionario. Es una técnica estructural o de interdependencia, lo que significa que todas las variables tienen la misma importancia y son independientes (Morales, 2011; García, Gill y 
Rodríguez, 2000; Garza, 2013). El objetivo de esta técnica es resumir la información para describirla más fácilmente; es decir, a partir de una serie de variables dadas se debe encontrar una estructura más simple que ayude a la construcción de nuevos conceptos y teorías. El análisis exploratorio está caracterizado porque no se conocen a priori el número de factores y es en la aplicación empírica donde se determina el número. Particularmente se pretende responder a la pregunta ¿Por qué unas variables se relacionan más con unas y menos con otras? La respuesta hipotética sería porque existen unas dimensiones en las cuales unas variables explican más la variabilidad común que en otras.

En el desarrollo de la técnica, Lloret-Segura, FerreresTraver, Hernández-Baeza y Tomás-Marco (2014), sugieren usar la matriz de correlaciones de Pearson si la escala tiene al menos 5 alternativas, así como también recomiendan usar la matriz policórica en el caso que las variables tengan una escala menor a 5. Aunque lo más recomendable hoy en día es revisar las distribuciones de cada ítem como paso previo y comparar ambos procedimientos porque también se ha comprobado que dichos procedimientos indican el mismo número de factores (Freiberg, Beatriz, De la Iglesia y Fernández, 2013).

En nuestro caso hemos optado por usar la matriz de correlación policórica porque la escala usada de 1 a 4 es ordinal, y tenemos la ventaja que la muestra supera los 200 individuos.

\section{RESULTADOS Y DISCUSIÓN}

En primer lugar, teníamos que asegurar que en la matriz de correlaciones existen suficientes correlaciones entre las variables para justificar el empleo del análisis factorial. Sólo la pregunta 13 presentó correlaciones inferiores a 0.3 , lo que indica que esta pregunta probablemente deba ser eliminada antes de usar el AFE.

\section{Figura No 1. Matriz de Correlaciones}

$\begin{array}{ccccccccc}6 & 0.433 & 0.506 & 0.257 & 0.402 & 0.543 & 1.000 & & \\ 7 & 0.577 & 0.548 & 0.405 & 0.415 & 0.554 & 0.578 & 1.000 & \\ 8 & 0.473 & 0.473 & 0.418 & 0.404 & 0.488 & 0.527 & 0.702 & 1.000 \\ 9 & 0.496 & 0.511 & 0.454 & 0.405 & 0.498 & 0.584 & 0.674 & 0.666 \\ 10 & 0.511 & 0.517 & 0.418 & 0.434 & 0.549 & 0.563 & 0.698 & 0.662 \\ 11 & 0.483 & 0.411 & 0.368 & 0.325 & 0.442 & 0.439 & 0.594 & 0.511 \\ 12 & 0.430 & 0.501 & 0.395 & 0.359 & 0.492 & 0.475 & 0.586 & 0.552 \\ 13 & 0.245 & 0.061 & 0.196 & 0.092 & 0.234 & 0.246 & 0.204 & 0.228 \\ 14 & 0.435 & 0.356 & 0.325 & 0.340 & 0.481 & 0.462 & 0.538 & 0.487\end{array}$


Un dato muy importante que nos proporciona la matriz de correlaciones es el determinante de dicha matriz es igual a 0.000003097303554 , este determinante es muy bajo, casi cero, esto es un buen indicador porque supone la existencia de correlaciones entre sí muy altas, por tanto, es posible la realización del análisis factorial.

El siguiente paso para usar la técnica del análisis factorial es la verificación de la prueba de esfericidad de Bartlett, la cual indica si la matriz de correlaciones es la identidad, para que sea posible la continuidad debe rechazarse esta hipótesis nula, en nuestro caso los valores obtenidos son: Bartlett'sstatistic $=7393.2$, grados de libertad $=378$ y el valor $\mathrm{P}=0.000010$, esta prueba está basada en el estadístico Chi-cuadrado, donde claramente se rechaza la hipótesis de identidad y podemos proseguir con el análisis.
Se analizó la segunda prueba que consiste en la medida de adecuación de la muestra KMO, la cual compara los valores de los coeficientes de correlación observados con los coeficientes parciales, este estadístico toma el valor entre 0 y 1 , y cuanto más cerca esté a 1 será más adecuada la continuidad de la técnica, en nuestros datos obtuvimos que Kaiser-Meyer-Olkin (KMO) test $=0.94928$ y de acuerdo a la escala de valoración, el resultado es excelente para la realización de la técnica.

Conviene acá determinar la medida de adecuación individual de cada variable, esto se realiza con la matriz anti-imagen donde los valores de la diagonal principal deben superar el valor de 0.5 y fuera de la diagonal deben de ser bajos. Existen 29 residuales, es decir, el $7 \%$, con valores superiores a 0.05 , lo que indica que este porcentaje es mínimo, generalmente no debe superar los dos dígitos.

Figura No 2. Matriz de Residuales

\begin{tabular}{r|r|r|r|r|r|r|r|r|r|r|r}
06 & .008 & .069 & & .042 & -.004 & -.017 & -.024 & -.001 & -.027 & -.023 & -.0 \\
21 & -.066 & .037 & .042 & & .014 & .008 & .019 & -.013 & -.027 & -.021 & .0 \\
01 & -.025 & -.008 & -.004 & .014 & & .062 & -.019 & -.022 & -.012 & -.012 & -.0 \\
13 & .012 & .007 & -.017 & .008 & .062 & & .020 & .009 & -.038 & -.003 & .0 \\
08 & .026 & -.019 & -.024 & .019 & -.019 & .020 & & .044 & -.038 & -.016 & .0 \\
30 & -.011 & .002 & -.001 & -.013 & -.022 & .009 & .044 & & .037 & -.009 & .0 \\
05 & -.002 & -.019 & -.027 & -.027 & -.012 & -.038 & -.038 & .037 & & .087 & -.0 \\
52 & .022 & -.015 & -.023 & -.021 & -.012 & -.003 & -.016 & -.009 & .087 & & .0 \\
35 & .033 & -.042 & -.006 & .022 & -.010 & .020 & .023 & .002 & -.019 & .009 & \\
09 & -.001 & -.005 & -.012 & .010 & -.003 & .009 & .009 & .010 & -.009 & -.008 & .0 \\
39 & -.013 & .044 & .053 & -.014 & .008 & -.030 & .004 & -.002 & -.035 & -.013 & -.0 \\
22 & -.064 & .011 & -.019 & -.008 & .022 & .009 & .005 & -.002 & .028 & -.035 & .0 \\
08 & .020 & -.062 & -.024 & .028 & -.068 & -.022 & .004 & .040 & .029 & .055 & -.0 \\
10 & .053 & -.016 & .009 & -.045 & .004 & .014 & -.060 & -.015 & .003 & .013 & -.0 \\
09 & -.001 & -.027 & -.011 & -.025 & .016 & .016 & .026 & -.007 & .003 & -.011 & .0 \\
23 & .059 & .003 & .021 & .018 & .021 & -.070 & -.003 & -.030 & -.014 & -.002 & -.0
\end{tabular}


Igual que los otros estadísticos, las comunalidades juegan un papel determinante en el uso de la técnica del análisis factorial, pues indican la proporción de varianza en común con el resto de variables. Grandes cantidades de comunalidades indican que la solución factorial extrae gran cantidad de varianza de una variable. Un valor frontera permitido para que la extracción factorial agrupe los factores adecuados es 0.40 . Variables por debajo de este valor debe discutirse su permanencia en la solución factorial. En la Tabla $\mathrm{N}^{\circ} 1$ se presentan que los ítems número tres y cuatro tienen comunalidades por debajo de 0.3 , por lo que será necesario valorar la extracción factorial primero con ellas y luego sin ellos para valorar la solución factorial resultante.

\section{Tabla No 1. Comunalidades}

\begin{tabular}{llllllll}
\hline P1 & 0.557 & P8 & 0.588 & $\mathbf{P 1 5}$ & 0.617 & P22 & 0.605 \\
P2 & 0.551 & P9 & 0.714 & P16 & 0.607 & P23 & 0.606 \\
P3 & 0.275 & P10 & 0.732 & P17 & 0.511 & P24 & 0.516 \\
P4 & 0.298 & P11 & 0.554 & P18 & 0.554 & P25 & 0.73 \\
P5 & 0.522 & $\mathbf{P 1 2}$ & 0.566 & $\mathbf{P 1 9}$ & 0.605 & $\mathbf{P 2 6}$ & 0.582 \\
P6 & 0.491 & $\mathbf{P 1 3}$ & 0.429 & $\mathbf{P 2 0}$ & 0.511 & $\mathbf{P 2 7}$ & 0.651 \\
P7 & 0.715 & $\mathbf{P 1 4}$ & 0.68 & $\mathbf{P 2 1}$ & 0.554 & $\mathbf{P 2 8}$ & 0.666 \\
\hline
\end{tabular}

Una vez superado todos los supuestos y condiciones para la aplicación del análisis factorial, se entra al aspecto más importante de la técnica, que consiste en la determinación de la cantidad de factores y el porcentaje de varianza que explican.

En este caso se usó el método de mínimos cuadrados no ponderados (ULS) que tiene la ventaja sobre los demás porque no es requerida la normalidad de las variables y puede usarse en variables ordinales. El procedimiento de extracción se realizó con el programa FACTOR versión 10.3.01, y la determinación del número de factores se realizó por dos métodos: el de varianza explicada, es decir, el scree test, y el de Promedios Parciales Mínimos (MAP en inglés) propuesto por Velicer (1976), este método que da una solución alternativa que emplea una matriz de correlaciones parciales.
Ninguno de los factores se extrae después que la media al cuadrado de correlación parcial alcanza un mínimo. Este enfoque da un punto de parada exacto, tiene una interpretación operativa directa, y puede aplicarse a cualquier tipo de método de extracción. Por ello se han empleado varios métodos, porque las soluciones en un análisis factorial deben de ser cuidadosamente analizadas si se van a usar posteriormente para un análisis factorial confirmatorio.

Tabla No 2. Extracción factorial ULS usando la varianza explicada

\begin{tabular}{|c|c|c|c|}
\hline Variable & $\begin{array}{l}\text { Varianza del } \\
\text { Eigenvalor }\end{array}$ & $\begin{array}{c}\text { Proporción } \\
\text { de varianza } \\
\text { explicada }\end{array}$ & $\begin{array}{l}\text { Proporción } \\
\text { acumulada }\end{array}$ \\
\hline 1 & 13.02609 & 0.52104 & 0.52104 \\
\hline 2 & 1.55604 & 0.06224 & 0.58329 \\
\hline 3 & 1.19303 & 0.04772 & 0.63101 \\
\hline 4 & 0.91903 & 0.03676 & \\
\hline 5 & 0.78449 & 0.03138 & \\
\hline 6 & 0.69746 & 0.0279 & \\
\hline 7 & 0.63633 & 0.02545 & \\
\hline 8 & 0.59216 & 0.02369 & \\
\hline 9 & 0.58655 & 0.02346 & \\
\hline 10 & 0.54711 & 0.02188 & \\
\hline 11 & 0.51262 & 0.0205 & \\
\hline 12 & 0.48645 & 0.01946 & \\
\hline 13 & 0.42795 & 0.01712 & \\
\hline 14 & 0.4107 & 0.01643 & \\
\hline 15 & 0.36073 & 0.01443 & \\
\hline 16 & 0.34691 & 0.01388 & \\
\hline 17 & 0.29091 & 0.01164 & \\
\hline 18 & 0.27472 & 0.01099 & \\
\hline 19 & 0.26946 & 0.01078 & \\
\hline 20 & 0.24136 & 0.00965 & \\
\hline 21 & 0.23253 & 0.0093 & \\
\hline 22 & 0.18231 & 0.00729 & \\
\hline 23 & 0.16596 & 0.00664 & \\
\hline 24 & 0.14514 & 0.00581 & \\
\hline 25 & 0.11396 & 0.00456 & \\
\hline
\end{tabular}

La primera solución obtenida basada en la varianza acumulada que supera el $60 \%$ está determinada por tres 
factores.AlmismotiempocoincidiríaconlaregladeKaiser, pero es una mera coincidencia. En la Tabla No 2 tenemos que el modelo de evaluación al desempeño docente que ha vertido la aplicación de nuestro cuestionario estaría compuesto por tres factores, los cuales explican el $63.1 \%$ de la varianza, porcentaje catalogado como muy bueno para la propuesta de un modelo.

Las comunalidades de las 25 variables todas tienen carga por encima de 0.4 , lo que indica que las variables originales están moderadamente correlacionadas. Lo más importante es observar que todas las variables tienen carga significativa. Por lo que procederemos a rotar la matriz para obtener una mejor interpretación de los factores.

Tabla No 3. Rotación de los factores con PROMAX

\begin{tabular}{|c|c|c|c|}
\hline & F1 & F2 & F3 \\
\hline $\mathrm{V} \quad 1$ & & 0.302 & 0.424 \\
\hline $\mathrm{V} 2$ & & 0.3 & 0.53 \\
\hline V 5 & & 0.496 & 0.346 \\
\hline $\mathrm{V} 6$ & & 0.465 & \\
\hline V 7 & & 0.874 & \\
\hline $\mathrm{V} 8$ & & 0.638 & \\
\hline $\mathrm{V} 9$ & & 0.628 & \\
\hline V 10 & & 0.733 & \\
\hline V 11 & & 0.891 & \\
\hline V 12 & & 0.785 & \\
\hline V 14 & & 0.795 & \\
\hline V 15 & & 0.487 & \\
\hline V 16 & & & 0.906 \\
\hline V 17 & & & 0.673 \\
\hline V 18 & 0.332 & & 0.424 \\
\hline V 19 & 0.52 & & 0.339 \\
\hline V 20 & 0.615 & 0.301 & \\
\hline V 21 & 0.413 & 0.48 & \\
\hline V 22 & 0.33 & 0.346 & \\
\hline V 23 & 0.345 & 0.346 & \\
\hline V 24 & 0.359 & 0.408 & \\
\hline V 25 & 0.699 & & \\
\hline V 26 & 0.797 & & \\
\hline V 27 & 0.815 & & \\
\hline V 28 & 0.501 & 0.444 & \\
\hline
\end{tabular}

En la tabla anterior tenemos la matriz factorial rotada. Se empleado la rotación oblicua que es la más recomendada porque se ha demostrado ampliamente que los factores que constituyen al desempeño docente están correlacionados, tal como lo podemos ver en la Tabla No 4, donde los tres factores están correlacionadas positivamente y con valores superiores a 0.6 , esto justifica la elección de la rotación oblicua sobre la ortogonal.

Tabla No 4. Correlación entre factores

\begin{tabular}{lllll}
\hline & \multicolumn{1}{c}{ F1 } & & F2 & F3 \\
\hline F1 & 1 & & & \\
F2 & 0.741 & 1 & & \\
F3 & 0.712 & 0.683 & 1 & \\
\hline
\end{tabular}

En la matriz rotada hemos omitido los valores inferiores a 0.3 en valor absoluto, luego para determinar a qué factor pertenece cada variable seleccionamos el valor más alto en valor absoluto. De este modo el primer factor está determinado por los ítems No 19, 20, 25, 26, 27, 28; el factor dos lo conforman los ítems No 5, $6,7,8,9,10,11,12,14,15,21,22,23,24$; finalmente el factor tres queda estructurado por los ítems No 1,2, $16,17,18$. Esta estructura tiene la ventaja que ningún factor quedó con menos de cinco ítems, por lo que de acuerdo con Izquierdo, Olea y Abad (2014) cumple satisfactoriamente la conformación de un factor.

$\begin{aligned} & \text { Tabla No 5. Fiabilidad de cada uno de factores } \\
& \text { obtenidos }\end{aligned}$
\begin{tabular}{lll}
\multicolumn{2}{c}{ Factor } & Fiabilidad \\
\hline 1 & 0.937 & \\
2 & 0.891 & \\
3 & 0.861 & \\
\hline
\end{tabular}

Cada uno de los tres factores presenta valores muy positivos en términos de fiabilidad y en el cuestionario en general se estima el alfa de Cronbach estandarizado en 0.9610 y el coeficiente omega en 0.9613 , valores considerados excelentes. 
Como los factores obtenidos no coinciden con la propuesta, se hace necesario definir operacionalmente el significado de las variables en cada uno de los factores obtenidos. Por tanto, se trabajará en este apartado usando el modelo de tres factores que a nuestro criterio es el más similar al planteamiento. El primer factor quedó compuesto por los ítems No 19 , 20, 25, 26, 27, 28; que está relacionado con el respeto hacia los alumnos y la interacción con los mismos.

El segundo factor lo conforman los ítems No 5, 6, $7,8,9,10,11,12,14,15,21,22,23,24$; este factor relaciona aspectos organizativos y dominio de la clase. Finalmente, el factor tres queda estructurado por los ítems No 1, 2, 16, 17, 18. Es evidente que este factor está vinculado con la planificación y cumplimiento.

\section{CONCLUSIONES}

Hay que destacar que en la matriz de correlaciones entre los factores obtenidos, los alumnos valoran el respeto y la interacción del maestro como el factor que más correlaciona positivamente con todos los aspectos de la práctica docente. Al mismo tiempo, los alumnos le dan mucha importancia a la forma en cómo los maestros organizan y desarrollan la clase, siendo el dominio, un aspecto elemental en la valoración obtenida. Es decir, los alumnos consideran que un docente se desempeña bien si es capaz de interactuar con respeto, organiza y cumple lo planificado, mostrando dominio en el desarrollo de sus actividades.

La segunda conclusión es que existen muchas variables relevantes asociadas a la práctica docente. En este caso se encontraron seis elementos que están determinando la evaluación del docente en la FAREM - Chontales, las cuales son: interacción del docente con el alumno, respeto del docente hacia el alumno, organización de la asignatura, dominio al impartir la asignatura, planificación de las actividades a desarrollar y cumplimiento de los deberes como docente.
El cuestionario llenado fue analizado con pruebas rigurosas sobre dimensionalidad, fiabilidad y constructo que permitieron encontrar tres dimensiones para todo el conjunto de variables contestadas por los estudiantes. Estas dimensiones se denominaron: interacción y respeto, organización y dominio, y, planificación y cumplimiento, todas ellas descritas anteriormente.

El último objetivo ha sido quizás el más complejo de realizar, pero a la vez el más novedoso. Existen una serie de procedimientos rigurosos y aún no definitivos para hablar de la estructura dimensional en un instrumento. Es decir, muchos investigadores elaboran una serie de preguntas y las dividen en lo que llaman factores, pero quizás esta estructura sea muy subjetiva, puesto que no se somete a pruebas estadísticas.

La misma unidimensionalidad es difícil de superar sino se construyen adecuados ítems con una escala apropiada, puesto que el mínimo debe superar el 50 $\%$ de la variabilidad de toda la escala. En este caso la aplicación del análisis factorial exploratorio permitió definir la multidimensionalidad del instrumento y conocer la estructura misma de dichas dimensiones. Se ha podido comprobar hasta dos veces la misma estructura, razón por la cual se tiene mucha confianza en los resultados obtenidos.

En el desarrollo de la técnica del análisis factorial exploratorio se ha visto que el cuestionario ha superado todos los supuestos teóricos y técnicos. La matriz de correlaciones permitió determinar que era viable realizarlo, que existían muchos ítems que presentaban buenos índices de discriminación y aquellos que no cumplían el requisito fueron excluidos del análisis.

La técnica y sus diferentes métodos usados para la extracción, sugieren que hay más de una dimensión en el cuestionario, tres dimensiones correlacionadas porque con la rotación oblicua dichas correlaciones han sido altas. Con esta investigación se ha acercado 
a una comprobación empírica de la estructura del cuestionario compuesta por variables no observadas con una importante relación entre ellas.

\section{REFERENCIAS BIBLIOGRÁFICAS}

Barroso, J. y cabero, J. (2010). La investigación educativa en TIC. Visiones

Cochran, W.G. 1977. Sampling Tecniques. 3d. ed., Wiley y Sons, New York

Cronbach, L. J. (1963). Course improvement throuhg evaluation. Teachers College Records(64), 672683.

Dias, J. (2000). Avaliacão da educacão superior. Petrópolis: Vozes.

Fernández, J. (2008). Valoración de la calidad docente: El profesorado. Un modelo de evaluación curricular. Madrid: Editorial Complutense.

Freiberg, A., Beatriz, J., De la Iglesia, G., y Fernández, M. (2013). Correlaciones policóricas y tetracóricas en estudios factoriales exploratorios y confirmatorios. Ciencias Psicológicas, VII(2), 151-164.

García Jiménez, E., Gill, J., y Rodríguez, G. (2000). Análisis Factorial. Madrid: La Muralla.

García Ramos, J. M. (1996). Valoración de la competencia docente del profesor universitario: una aproximación empírica. Revista Complutense de Educación, 8(2), 81-108.

Garza, J. d. (2013). Análisis estadístico multivariante: un enfoque práctico. Ciudad de México: McGraw Hill.

Hayman, J. L. (1984). Investigación y educación. Barcelona: Paidos.

Hernández Sampieri, R., Collado, C. F., y Baptista, M. d. (2014). Metodología de la Investigación (Sexta ed.). México: McGraw-Hill.

Izquierdo, I., Olea, J., y Abad, F. (2014). Exploratory factor analysis in validation studies: Uses and recommendations. Psicothema, 26(3), 395-400. doi:10.7334/psicothema2013.349

Lloret-Segura, S., Ferreres-Traver, A., Hernández-
Baeza, A., y Tomás-Marco, I. (2014). El análisis factorial exploratorio de los ítems: una guía práctica, revisada y actualizada. anales de psicología, 30(3), 1151-1169.

Marsh, H. W. (1984). Student's evaluation of university teaching:Dimensionality reliability, validity, potential biases, and utility. Journal of EducationalPsychology, 76, 707-754.

Mateo, J. (1988). La evaluación del Profesorado Universitario; algunas Consideraciones Respecto al Estado de la Cuestión. Valencia: Universidad de Valencia.

Mateo, J.(1998). La evaluación educativa. Enciclopedia General de la Educación, 532-586.

Morales, J. J. (2001). La evaluación en el área de Educación Visual y Plástica en la ESO. Barcelona: NARCEA.

Morón, J. A. (1999). Las cualidades de los docentes según los alumnos. Sevilla: Diputación de Sevilla.

Pérez, C. (2005). Muestreo Estadístico: Conceptos y problemas resueltos. Madrid: Pearson Educación.

Piaget, J. (1969). Psicología y Pedagogía. Paris: Critica.

Romero Díaz, T. (2014). Cuestionario de opinion para la evaluación del desempeño docente en la UNANMANAGUA, Nicaragua, FAREM-Chontales. Actualidades Investigativas en Educación, 14(2), 269-297.

Romero Díaz, T., y Martínez, A. (2017). La construcción de instrumentos de evaluación del desempeño docente en la Universidad desde un enfoque cualitativo. Revista Universitaria del CARIBE, 18(1), 15-27.

Rueda, M. (2011). La evaluación de los docentes, elemento sustantivo en la educación. Perfiles Educativos, 33 (133), 1-7.

Salazar, J. (2008). Diagnóstico preliminar sobre evaluación de la docencia universitaria. Una aproximación a la realidad en las universidades públicas $\mathrm{y} / \mathrm{o}$ estatales de Chile. Revista Iberoamericana de Evaluación Educativa, I-III, 67-84. 
Scriven, M. (1994). Evaluation as a discipline. Studies in Educational Evaluation, 20, 147-166.

Stake, R. E. (1982). Conversando sobre avaliacão. En M. A. Goldberg, y C. P. Souza, Avalicão de programas Educacionais: vicissitudes, controvérsias, desafios (págs. 30-34). Sao Paulo: EPU.

Stufflebeam, D. L. (1995). The Personnel Evaluation Standards: How to assess Systems for Evaluating Educators (Sixth ed.). California: Corwin Press.

Tyler, R. W. (1969). Basic Principles of Curriculum and Instruction. Chicago: University of Chicago Press. Valdés, H. (2000). Encuentro Iberoamericano sobre Evaluación del Desempeño Docente. Ciudad de México: CICE.

Valderrey, P. (2010). SPSS 17: Extracción del conocimiento a partir del análisis de datos. Madrid: Alfaomega.
Viana, H. M. (2000). Avaliacão educacional: teoria, planejamento e modelos. São Paulo: IBRASA.

Velicer, W. (1976). Determining the number of components from the matrix of partial correlations. Psychometrika(41), 321-327.

Urriola, K. M. (2013). Sistema de evaluación del desempeño profesional docente aplicado en Chile. Percepciones y vivencias de los implicados en el proceso. El caso de la ciudad de Concepción. (Tesis doctoral inédita). Departamento de Didáctica y Organización Educativa. Universitat de Barcelona. Zambrano, A. (2014). Prácticas evaluativas para la mejora de la calidad del aprendizaje: Un estudio contextualizado en La Unión - Chile. (Tesis inédita). Departamento de Pedagogía Aplicada. Universitat Autónoma de Barcelona. 